\title{
SALUD Y CALIDAD DE VIDA EN EL ADULTO MAYOR
}

\section{HEALTH AND QUALITY OF LIFE INTHE ELDERLY}

\author{
Luis F. Varela Pinedo", a
}

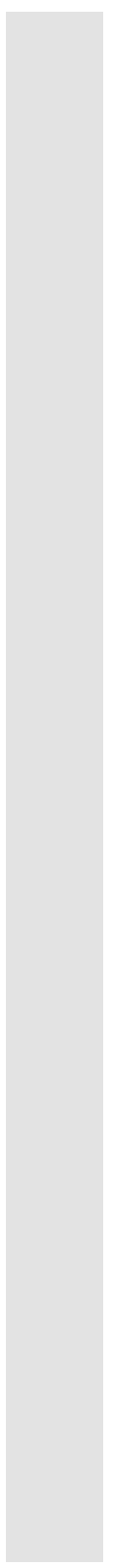

\begin{abstract}
El envejecimiento poblacional es un fenómeno no exclusivo de países desarrollados, donde los mayores de 60 años alcanzan tasas de 15 a $20 \%$ de la población general ${ }^{(1)}$; sino que también ocurre en nuestro país. De acuerdo con el Instituto Nacional de Estadística e Informática (INEI), en el año 2015 la población mayor de 60 años representó el 10\%, siendo los mayores de 80 años el grupo con mayor tasa de crecimiento ${ }^{(2)}$. Además, estimaciones poblacionales indican que para el año 2025 las personas adultas mayores constituirán entre el 12 al $13 \%$ de la población peruana ${ }^{(2,3)}$. Este crecimiento se desarrollará en un contexto social particular que es desfavorable para los adultos mayores y una transición epidemiológica caracterizada por un aumento en la prevalencia de las enfermedades crónicas no transmisibles. Cabe señalar que la Organización Mundial de la Salud (OMS) considera como adulta mayor a toda persona mayor de 60 años ${ }^{(4)}$.
\end{abstract}

Como respuesta al envejecimiento poblacional, la Asamblea General de las Naciones Unidas convocó, en los años 1982 y 2002, a asambleas mundiales para tratar este problema. En dichas reuniones se estableció un compromiso mediante una declaración política y un plan de acción internacional que incorpora tres orientaciones: las personas de edad y el desarrollo, el fomento de la salud y bienestar en la vejez, y la creación de un entorno propicio y favorable ${ }^{(4,5)}$.

En concordancia con los lineamientos dados en la Primera Asamblea Mundial sobre el Envejecimiento, en el año 2000 se conformó en el Perú una comisión multisectorial para la formulación del Plan Nacional de las Personas Adultas Mayores (PLANPAM), que desde el 16 de junio de 2013 cuenta con el PLANPAM 2013-2017: "Promoviendo el envejecimiento activo y saludable", el cual está vigente y cuyo objetivo es mejorar la calidad de vida de las personas adultas mayores; todo ello basado en cuatro políticas nacionales esenciales: a). envejecimiento saludable; b). empleo, previsión y seguridad social; c). participación e integración social, y d). educación, conciencia y cultura sobre el envejecimiento y la vejez. Este plan ha sido elaborado considerando los enfoques básicos que orientan la acción del sector: de derechos humanos, de igualdad de género, de interculturalidad, así como el enfoque intergeneracional (6).

La doctora Margaret Chan, directora general de la OMS, en el "Informe mundial del envejecimiento y la salud" publicado el año 2015, menciona las profundas consecuencias para la salud y para los sistemas de salud, su personal y su presupuesto, que implicará el envejecimiento poblacional; señala también que los desafíos planteados en el presente siglo deben ser enfrentados con prontitud. En el informe se enfatiza que en la mayoría de personas mayores lo más importante es mantener la capacidad funcional, y que el envejecimiento saludable es algo más que la ausencia de enfermedad. Se pretende, luego de un debate multidimensional, encontrar una respuesta más apropiada de la salud pública respecto al envejecimiento de la población; es decir, considerar esta etapa como una nueva oportunidad para las personas y la sociedad ${ }^{(5)}$.

La OMS, desde 1948, ha definido la salud como: Un estado de completo bienestar físico, mental y social, no solamente la ausencia de enfermedad o dolencia. Esta definición ha sido cuestionada, ya que se considera como una situación ideal, difícilmente alcanzable por toda la población. Machteld

\footnotetext{
Instituto de Gerontología, Universidad Peruana Cayetano Heredia. Lima, Perú.

Médico internista, geriatra, doctor en Medicina, editor invitado.

Recibido: 07/03/2016 Aprobado: 23/03/2016
}

Citar como: Varela Pinedo LF. Salud y calidad de vida en el adulto mayor. Rev Peru Med Exp Salud Publica. 2016;33(2):199-201. doi: 10.17843/rpmesp.2016.332.2196 
Huber et al., en el artículo titulado: "How should we define health", consideran que la definición de salud planteada por la OMS no es aplicable en el contexto de la transición demográfica-epidemiológica, y del aumento de la prevalencia de enfermedades crónicas. Estos autores sostienen que se debe enfatizar la habilidad de adaptación de las personas, por sí mismas, en el aspecto social, físico y emocional ${ }^{(7)}$.

En el PLANPAM 2013-2017 se refiere al envejecimiento saludable como: La mejora del bienestar físico, psíquico y social de las personas adultas mayores se logra a través del incremento en la cobertura y calidad de los servicios sociosanitarios, la garantía de una asistencia alimentaria y nutricional, la generación de oportunidades para una vida armónica dentro de su familia y su comunidad ${ }^{(6)}$.

La OMS considera al envejecimiento activo como: El proceso de optimización de las oportunidades de salud, participación y seguridad con el fin de mejorar la calidad de vida a medida que las personas envejecen ${ }^{(8)}$. El envejecimiento exitoso es un estado donde un individuo es capaz de invocar mecanismos adaptativos psicológicos y sociales para compensar limitaciones fisiológicas, para lograr una sensación de bienestar y una alta autoevaluación de la calidad de vida y un sentido de realización personal, incluso en el contexto de la enfermedad y discapacidad ${ }^{(9)}$

La calidad de vida se relaciona a factores económicos, sociales y culturales en el entorno que se desarrolla $y$, en gran medida, a la escala de valores de cada individuo. En la década de los noventa se desarrolló el concepto de calidad de vida vinculado a la salud, que se define como: El valor asignado a la duración de la vida, modulado por las limitaciones, estado funcional, percepciones y oportunidades sociales, y que se ven influidas por las enfermedades, lesiones, tratamientos y políticas sanitarias ${ }^{(10)}$. Existe, por tanto, consenso en que la calidad de vida deber ser considerada desde una visión multidimensional, que además de lo anteriormente mencionado, debe incluir aspectos ambientales, culturales, económicos, de servicios de salud, satisfacción, actividades de ocio, entre otros ${ }^{(11)}$.

En el estudio: "Perfil clínico, funcional y sociofamiliar del adulto mayor de la comunidad en un distrito de Lima, Perú", recientemente publicado en la Revista Peruana de Medicina Experimental y Salud Pública, se encuentra que de 497 personas encuestadas, solo el $23 \%$ tuvo la autopercepción de considerar su salud como buena o muy buena, y cerca al $60 \%$ tenían riesgo o problema social ${ }^{(12)}$.

Por el crecimiento constante y las características del envejecimiento poblacional de los países en desarrollo, el Programa de Salud y Envejecimiento de la OMS consideró necesario investigar las condiciones de salud de la población adulta mayor en este tipo de países. Para lo cual, diseñó el estudio "Desarrollando respuestas integradas de los sistemas de cuidados de la salud para una población en rápido envejecimiento" que se ejecutó en 18 países, siendo el nuestro uno de los participantes. Uno de los resultados fue que en el Perú, una de las razones por la que la población adulta mayor no acudía a los centros de atención primaria en salud era por sus escasos recursos económicos ${ }^{(13)}$. En la actualidad se ha mejorado el acceso de las personas adultas mayores a los servicios del Ministerio de Salud, debido al Seguro Integral de Salud (SIS).

Es importante mencionar que Emily Greenfield considera que la creación y mantenimiento de comunidades amigables con la edad y el envejecimiento saludable, son una vía para dar soporte de salud, vigor y una convivencia en armonía para el adulto en la comunidad ${ }^{(14)}$.

En este número de la Revista se presenta, el simposio "Hacia una atención integral de la salud del adulto mayor", en concordancia con la visión que se debe tener hacia este grupo etario, se ha invitado a diferentes especialistas: geriatras, internistas, neurólogos, psiquiatras y especialistas en medicina familiar.

"La promoción de la salud y prevención de las enfermedades para un envejecimiento activo y con calidad de vida", tiene como finalidad, dar las pautas para el logro de un envejecimiento exitoso, con la menor cantidad de enfermedades posibles o adecuadamente controladas y manteniendo autonomía, tema presentado por las doctoras Elizabeth Aliaga, Marcela Mar y Sofía Cuba.

Los doctores Miguel Gálvez, Helver Chávez y la doctora Elizabeth Aliaga en el artículo que presentan sobre "Valoración geriátrica integral: utilidad en la evaluación de la salud del adulto mayor" resaltan la utilidad que esta tiene en la evaluación, identificación y priorización de los aspectos negativos y positivos de la salud de las personas adultas mayores, con el fin de prevenir y tratar la enfermedad, rehabilitar la discapacidad e impulsar un envejecimiento activo y saludable.

La identificación de la fragilidad en los adultos mayores predice eventos adversos como caídas, hospitalización, discapacidad y muerte. El manejo de este problema se presenta en el artículo "Fragilidad en el adulto mayor: detección, intervención en la comunidad y toma de decisiones en el manejo de enfermedades crónicas", presentado por la doctora Tania Tello y el doctor Luis Varela.

"Salud mental en el adulto mayor: trastornos neurocognitivos mayores, afectivos y del sueño", tema presentado por las doctora Tania Tello y los doctores Renato Alarcón y Darwin Vizcarra, donde se enfatiza la identificación temprana de dichos problemas, con la finalidad de realizar una intervención oportuna 
La prescripción adecuada de fármacos basada en evidencia para disminuir la medicación inapropiada, la polifarmacia y la automedicación, es tratada en el tema de "Estrategias para optimizar el manejo farmacológico en el adulto mayor", cuya presentación está a cargo de los doctores Pedro Ortiz, Eduardo Penny y la doctora Paola Casas.

Un análisis de los modelos, niveles de atención, coordinación y planificación de diferentes servicios de asistencia de salud a la población adulta mayor se presenta en el artículo "Atención sociosanitaria de los adultos mayores en el Perú" a cargo de los doctores Helver Chávez, Juan Del Canto y la doctoras Rossana Apaza y Paola Casas.
Como se ha mencionado, la concepción de salud, envejecimiento saludable, exitoso, activo y calidad de vida de las personas adultas mayores, debe ser abordada de una manera integral, en la que, además del enfoque del aspecto de salud, se considere el bienestar personal, su funcionalidad y los entornos sociofamiliares propicios y favorables que les permita continuar su desarrollo personal. Todo ello parte de una decisión política de implementación y ejecución de las normas ya promulgadas y que deberían conducir al desarrollo de una cultura pertinente sobre el envejecimiento y la vejez, en donde la persona adulta mayor debe ser el actor principal.

\section{REFERENCIAS BIBLIOGRÁFICAS}

1. Varela L, Chávez $\mathrm{H}$, Gálvez $\mathrm{M}$, Méndez F. Funcionalidad en el adulto mayor previo a su hospitalización a nivel nacional. Rev Med Hered. 2005;16(3):165-71.

2. Instituto Nacional de Estadística e Informática. Estado de la población peruana 2015. Lima: INEI; 2015. Disponible en: http://www.inei. gob.pe/media/MenuRecursivo/ publicaciones_digitales/Est/Lib1251/ Libro.pdf

3. Instituto Nacional de Estadística e Informática. Perú: Estimaciones y Proyecciones de Población, 19502050. Lima: INEI; 2009. Disponible en: http://proyectos.inei.gob.pe/ web/biblioineipub/bancopub/Est/ Lib0845/index.htm

4. Varela L, Tello T. Asambleas mundiales sobre el envejecimiento. En: Varela L. Principios de Geriatría y Gerontología. 2da ed: Lima: Universidad Peruana Cayetano Heredia; 2011. p 19-24.

5. Organización Mundial de la Salud. Informe mundial sobre el envejecimiento y la salud. Ginebra: OMS; 2015. Disponible en: http://apps.who.int/iris/bitstre $\mathrm{am} / 10665 / 186466 / 1 / 9789240694873$ spa.pdf

6. Perú, Ministerio de la Mujer y Poblaciones Vulnerables. Plan nacional de personas adultas mayores 2013-2017. Cuadernos sobre poblaciones vulnerables $\mathrm{N}^{\circ}$ 5. Lima: MINP; 2013. Disponible en: http://www.mimp.gob.pe/files/ mimp/especializados/boletines_dvmpv/ cuaderno_5_dvmpv.pdf

7. Huber M, Knottnerus JA, Green L, van der Horst H, Jadad AR, Kromhout D, et al. How should we define heath?. BMJ. 2011 Jul 26;343:d4163. doi: 10.1136/ bmj.d4163.

8. Organización Mundial de la Salud. Envejecimiento activo: un marco político. Rev Esp Geriatr Gerontolol. 2002;37(S2): 74-105.

9. Young Y, Frick KD, Phelan EA. Can successful aging and chronic illness coexist in the same individual? A multidimensional concept of successful aging. J Am Med Dir Assoc. 2009
Feb;10(2):87-92. doi: 10.1016/j. jamda.2008.11.003.

10. Paterne G, Bardales Y, Martinez M. Calidad de vida. En: Abizanda P. Medicina Geriátrica. Barcelona: Elsevier; 2012. p. 155-62.

11. Lisigurski M, Varela L. Calidad de vida. En: Varela L. Principios de Geriatría y Gerontología. Lima: Universidad Peruana Cayetano Heredia; 2003. p. 63-7.

12. Varela L, Chávez H, Tello T, Ortiz P, Gálvez M, Casas P, et al . Perfil clínico, funcional y sociofamiliar del adulto mayor de la comunidad en un distrito de Lima, Perú. Rev Peru Med Exp Salud Publica. 2015;32(4):709-15.

13. Varela L. Desarrollando respuestas integradas en los sistemas de salud de una población en rápido envejecimiento. INTRA III. Lima: UPCH-OPS/OMS; 2005.

14. Greenfield E. Healthy aging and agefriendly community initiatives. Public Policy \& Aging Report, 2015;25:43-6.

\section{Nuestros artículos se encuentran indizados en:}

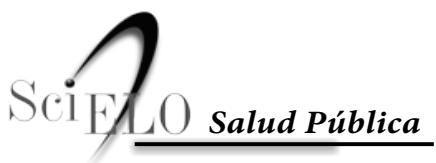

www.scielo.org 